\title{
A model of financial market dynamics with heterogeneous beliefs and state-dependent confidence
}

\author{
Carl Chiarella (carl.chiarella@uts.edu.au) \\ School of Finance and Economics, University of Technology Sydney, Australia \\ Roberto Dieci*(rdieci@rimini.unibo.it) \\ Department of Mathematics for Economic and Social Sciences \\ University of Bologna, Italy \\ Laura Gardini (gardini@econ.uniurb.it) \\ Institute of Economics, University of Urbino, Italy \\ Lucia Sbragia (l.sbragia@uniurb.it) \\ Institute of Economics, University of Urbino, Italy
}

\begin{abstract}
In a simple model of financial market dynamics, we allow the price of a risky security to be set by a market maker depending on the excess demand of heterogeneous interacting traders, fundamentalists and chartists, who place their orders based upon different expectations schemes about future prices: while chartists rely on standard trend-based rules, fundamentalists are assumed to know the economic environment and to form their beliefs accordingly. As price moves away from the long-run fundamental, fundamentalists become less confident in their forecasts, and put increasing weight on a reversion towards the fundamental price. The resulting two-dimensional discrete time dynamical system can exhibit a rich range of dynamic scenarios, often characterized by coexistence of attractors. A simple noisy version of the model reveals a variety of possible patterns for return time series.
\end{abstract}

Keywords: heterogeneous beliefs, financial market dynamics, bifurcation analysis, coexisting attractors

JEL classification: C62, D84, E32, G12

\section{Introduction}

The literature on the dynamics of financial prices arising from the interaction of heterogeneous agents has become well-developed over the last two decades, as recently discussed in two surveys by Hommes (2006) and LeBaron (2006). The common setup of a number of heterogeneous agent models is represented by a stylized market with one risky asset and one riskless asset, and the main focus is on the effect of heterogeneous beliefs and trading rules

\footnotetext{
${ }^{*}$ Corresponding author. Mailing address: Università degli Studi di Bologna, Facoltà di Economia del Polo di Rimini, Via D. Angherà 22, I-47900 Rimini, Italy. Phone +39 (0541) 434140
} 
on the dynamics of the price and return of the risky security. On the one hand, this literature shows how the interaction of heterogeneous agents can produce sustained deviations of financial prices away from their fundamentals; on the other hand, such models are able to reproduce the essential characteristics of empirical return time series and distributions. Both these features appear to be strictly related to the nonlinear laws of motion that often characterize such heterogeneous agent models, and to their interaction with exogenous noise processes (such as noisy fundamental/dividend process, or noisy demand components). Most models however result in high dimensional dynamical systems, that are of necessity not very tractable analytically, or may include too many parameters, which makes it difficult to test the robustness of simulation results. Within this heterogeneous agents literature, a particular role is played by models of fundamentalists-chartists interaction, which are able to capture a basic mechanism of price fluctuations, namely the interplay between the destabilizing forces of trend-chasing strategies and the stabilizing role of fundamental traders, who place their orders betting on mean reversion towards fundamentals. From a mathematical point of view, what prevents prices from diverging under the pressure of strong trend extrapolation - by replacing an unstable fundamental equilibrium with other stable attractors - is some nonlinear mechanism of adjustment, or interaction. In Day and Huang (1990) nonlinearity is related to fundamentalists' perceived chance for capital gains and losses. In Chiarella (1992), nonlinearity is due to a portfolio adjustment argument, that imposes nonlinear asset demand functions. In the model proposed by Brock and Hommes (1998) and further developed by Chiarella and He (2001, 2003), Hommes et al. (2005), agents switch between costly rational and cheaper naive prediction rules according to a nonlinear mechanism based upon realized profits. In a sense, these nonlinear factors play a role which is similar to the so-called jump variable technique, which avoids divergent paths within rational expectations models. The traditional argument justifying this technique is that agents realize that the movement away from equilibrium cannot last forever. Following a similar idea, in this paper we develop a simple model of fundamentalists and chartists, where the agents' belief that periods of increasing deviations from equilibrium must eventually come to an end is the only reason for stabilizing market forces to come into play and to bring prices back to long-run fundamentals, without the introduction of any further nonlinear effects. The idea used in this paper is similar (but even simpler) to the one developed in Chiarella and Khomin (2000) within a model of monetary dynamics with inflationary expectations.

In addition, the proposed model results in only a two-dimensional dynamical system which allows some analytical tractability - and is based upon a relatively low number of parameters.

We consider a simple model of financial market dynamics with two groups of heterogeneous traders, 'rational' fundamentalists and chartists, who adopt different expectations schemes in order to predict the price of the risky asset and to form their demand. A market maker is assumed to clear the market at the end of each trading period and to adjust the asset price in the direction of the excess demand. While chartists only use past price time series to form their expectations, fundamental traders are assumed to be informed about their economic environment, including other agents' behaviour, and to use their knowledge in formulating their forecast about next period price. However, their degree of confidence in such a forecast is state-dependent, in that they anticipate that the further the price deviates from its longrun equilibrium (fundamental) value, the more likely is it to revert towards equilibrium; 
as a consequence they put increasing weight on their belief of mean reversion as the price increasingly deviates from the fundamental. Our analysis shows that the simple interaction mechanism of the present model is able to generate a wide range of dynamic scenarios and time patterns for the price and return of the risky asset.

The plan of the paper is as follows. In section 2 we introduce the asset price dynamics under heterogeneous beliefs, discuss the expectations scheme used by fundamentalists and chartists (2.1) and express the dynamic model as a discrete time noisy dynamical system (2.2). Section 3 focuses on the properties of the two-dimensional map which represents the deterministic 'skeleton' of the dynamic model, in particular the fixed point and its stability properties (3.1), and the symmetry of the map (3.2). In section 4 we analyse by numerical simulations the dynamic behavior of the system when the parameters are chosen "outside" the stability domain, and show that the long-run dynamic scenario is quite often represented by coexistence of attractors, with the phase-space shared amongst different basins of attraction: in particular, sections 4.1 and 4.2 explore the behavior of the system in presence of contrarians and trend followers, respectively, while section 4.3 suggests how different deterministic scenarios may interact with exogenous noise on demand and dividends to generate time series with qualitatively different patterns and distributional characteristics. Section 5 concludes, while mathematical details are provided in the Appendices.

\section{The model}

The model setup follows Brock and Hommes (1998), Chiarella and He (2001, 2003) and Hommes et al. (2005). We consider a stylized financial market model with one risky asset and one risk free asset. The latter yields a gross return $R=1+r$, where $r>0$ is the constant risk free rate (per trading period). Let $p_{t}$ be the price (ex dividend) per share of the risky asset at time $t$ and $\left\{y_{t}\right\}$ be the stochastic dividend process. Assuming $H$ heterogeneous groups of agents, the wealth of investor of type $h(h=1,2, \ldots, H)$ evolves according to

$$
W_{h, t+1}=R W_{h, t}+z_{h, t}\left(p_{t+1}+y_{t+1}-R p_{t}\right),
$$

where $W_{h, t}$ is investor's wealth at time $t$ and $z_{h, t}$ is the number of shares he/she holds from $t$ to $t+1$. Let $E_{h, t}$ and $V_{h, t}$ denote the conditional expectation and variance of type- $h$ trader. Dividends are assumed to be i.i.d. in agents' beliefs, with $\bar{y}=E_{h, t}\left(y_{t+1}\right), h=$ $1,2, \ldots, H$, denoting the (commonly shared) expectation of the dividend. Denote also by $R_{t+1}:=p_{t+1}+y_{t+1}-R p_{t}$ the excess return per share in the trading period $(t, t+1)$. Assume that traders are expected utility maximizers, with exponential utility of wealth function $U_{h}(W)=-\exp \left(-a_{h} W\right)$, where $a_{h}$ is the risk aversion coefficient of type $h$-trader. Then, under the standard conditional normality assumption, the demand $z_{h, t}$ of type- $h$ trader for the risky asset is given by

$$
z_{h, t}=\frac{E_{h, t}\left(R_{t+1}\right)}{a_{h} V_{h, t}\left(R_{t+1}\right)} .
$$

In this paper we assume that there are two types of investors, called fundamental traders (or fundamentalists) and chartists, corresponding to type 1 and type 2 , and we denote by $n_{1}$ and $n_{2}=1-n_{1}$ their fixed market fractions ${ }^{1}$, respectively. A noisy component of demand

\footnotetext{
${ }^{1}$ Of course we could enrich the model (and its resulting price and return dynamics) by assuming timevarying market fractions, according to the evolutionary switching mechanism for expectation rules introduced
} 
is also considered, determined for instance by noise traders, captured by the term $\sigma_{\epsilon} \epsilon_{t}$, $\sigma_{\epsilon} \geq 0$, where $\left\{\epsilon_{t}\right\}$ is i.i.d. with zero mean and unit variance. Assuming zero supply of outside shares, the population-weighted average excess demand at time $t, z_{e, t}$, is thus given by $z_{e, t}=n_{1} z_{1, t}+n_{2} z_{2, t}+\sigma_{\epsilon} \epsilon_{t}$, or

$$
z_{e, t}=n_{1} \frac{E_{1, t}\left(R_{t+1}\right)}{a_{1} V_{1, t}\left(R_{t+1}\right)}+n_{2} \frac{E_{2, t}\left(R_{t+1}\right)}{a_{2} V_{2, t}\left(R_{t+1}\right)}+\sigma_{\epsilon} \epsilon_{t}
$$

The market price in each trading period is set by a market maker who takes a long (short) position when $z_{e, t}<0\left(z_{e, t}>0\right)$ in order to clear the market, and adjusts the price in the direction of the excess demand. The market maker price setting rule is thus

$$
p_{t+1}=p_{t}+\mu z_{e, t}
$$

where $\mu>0$ denotes the speed of price adjustment.

\subsection{Heterogeneous expectations}

In order to close the model, we must specify how fundamentalists and chartists adopt different schemes in order to form and update their beliefs about next period price. While the chartists follow a simple trend-based rule, the fundamentalists are assumed to be informed about their economic environment, including the behavior of other agents; in addition, they believe that the price is subject to mean reverting forces towards the long-run fundamental. More precisely, we model agents' expectations as follows:

(a) Fundamentalists expectation of next period price is given by

$$
E_{1, t}\left(p_{t+1}\right)=\alpha_{t} p_{t+1}^{e s t}+\left(1-\alpha_{t}\right) \bar{p}
$$

where $\bar{p}=\bar{y} /(R-1)$ is the long-run fundamental price ${ }^{2}$, while $p_{t+1}^{\text {est }}$ (to be specified later) represents the prediction based on their assumed superior information. By rewriting (5) as

$$
E_{1, t}\left(p_{t+1}-p_{t}\right)=\alpha_{t}\left(p_{t+1}^{e s t}-p_{t}\right)+\left(1-\alpha_{t}\right)\left(\bar{p}-p_{t}\right)
$$

one can view the fundamentalists as partially confident in a price movement in the direction of the predicted price $p_{t+1}^{e s t}$, and partially confident in a mean reversion towards the long-run fundamental. The weights of these components are state dependent: the further the price deviates from the fundamental $\bar{p}$, the more likely the fundamentalists believe that the price will revert towards $\bar{p}$. To capture such behaviour $\alpha_{t}$ is modelled as

$$
\alpha_{t}=\exp \left[-\frac{\left(\bar{p}-p_{t}\right)^{2}}{s}\right]
$$

where the parameter $s>0$ determines the sensitivity of fundamentalists with respect to the observed "mispricing": the lower $s$, the more weight fundamentalists put on mean reversion for any given deviation from the fundamental.

by Brock and Hommes (1997) within a cobweb framework, and further applied to an asset pricing model in the follow-up paper Brock and Hommes (1998). However, in this paper we want to focus purely on the effect of the mechanism we describe in the subsequent discussion.

${ }^{2}$ The fundamental price is computed via the usual discounted dividend formula. 
Put differently, though the fundamentalists hold an estimate based on their knowledge of the economic environment, their degree of confidence in this estimate (captured by the state dependent quantity $\alpha_{t}$ ) decreases in the presence of large price deviations from the fundamental price, when confidence in mean reversion dominates. A possible justification for this assumption is the experience of fundamentalists that in the past such long deviations from the fundamental have always finally reversed themselves. The fundamentalists also realise that large price deviations cannot last for a long time, due to the existence of limits to long/short positions of traders, and therefore they may want to reverse their positions before chartists do so. The assumed superior knowledge of the economic environment on the part of the fundamentalists gives them some edge in "getting out" before the chartists. This way of modelling "state-dependent confidence" of informed traders has already been used by Chiarella and Khomin (2000) in a different context, namely a model of monetary dynamics with inflationary expectations formed as a weighted average of heterogeneous beliefs ${ }^{3}$.

Thus for the fundamentalists, the expected excess return (per share) turns out to be

$$
E_{1, t}\left(R_{t+1}\right)=\bar{p}\left(R-\alpha_{t}\right)+\alpha_{t} p_{t+1}^{e s t}-R p_{t} .
$$

Substitution of eq. (7) into eq. (3), allows to interpret our model in a different fashion: it can be viewed as a naïve way of introducing endogenously varying proportions of two further groups of agents, within the goup of 'fundamental traders', namely rational and 'forward looking' agents who have full knowledge of the model structure ${ }^{4}$, whose proportion is $n_{1} \alpha_{t}$, and 'real' fundamentalists who expect mean reversion, whose fraction is $n_{1}\left(1-\alpha_{t}\right)$ : the higher the perceived mispricing, the larger is the fraction of traders who decide to bet on mean reversion towards the fundamental price. Note that under this interpretation our heterogeneous agent economy turns out to be made up of three different agent-types, one of which is the rational agent in the traditional sense.

(b) Chartists compute their expected price by extrapolating the most recent price change according to

$$
E_{2, t}\left(p_{t+1}\right)=p_{t}+g\left(p_{t}-p_{t-1}\right),
$$

and they are denoted as trend followers if $g \geq 0$, contrarians if $g<0$. It follows that

$$
E_{2, t}\left(R_{t+1}\right)=(1+g-R) p_{t}-g p_{t-1}+\bar{p}(R-1) .
$$

For simplicity we assume constant second moment beliefs for both agent-types, with $V_{1, t}\left(p_{t+1}\right)=\sigma_{1}^{2}, V_{2, t}\left(p_{t+1}\right)=\sigma_{2}^{2}$, as well as common beliefs about the variance of the i.i.d. dividend process, $V_{h, t}\left(y_{t+1}\right)=\sigma_{y}^{2}, h=1,2$. Therefore we obtain

$$
V_{h, t}\left(R_{t+1}\right)=\sigma_{h}^{2}+\sigma_{y}^{2}, \quad h=1,2
$$

\footnotetext{
${ }^{3}$ A similar mechanism to model endogenously varying weight of mean reverting forces has also been used by De Grauwe et al. (1993) within a heterogeneous agent model of exchange rate dynamics, though the model setup is quite different from ours and this weighting effect has a different economic motivation.

${ }^{4}$ Of course, one should assume that the estimate $p_{t+1}^{e s t}$ of 'forward looking' traders has been obtained at some cost, though we neglect information costs here because we don't model profits explicitly. These costs should be explicitly taken into account, however, if the switching among different predictors were based on realized profits, as in Brock and Hommes (1998).
} 


\subsection{The dynamical system}

Using (7), (8), (9), the fundamentalist and chartist demand functions are rewritten as

$$
z_{1, t}=\frac{\bar{p}\left(R-\alpha_{t}\right)+\alpha_{t} p_{t+1}^{e s t}-R p_{t}}{a_{1}\left(\sigma_{1}^{2}+\sigma_{y}^{2}\right)}, \quad z_{2, t}=\frac{(1+g-R) p_{t}-g p_{t-1}+\bar{p}(R-1)}{a_{2}\left(\sigma_{2}^{2}+\sigma_{y}^{2}\right)},
$$

while the price setting equation (4) becomes

$$
\begin{aligned}
p_{t+1}= & p_{t}+\mu\left\{A_{1}\left[\bar{p}\left(R-\alpha_{t}\right)+\alpha_{t} p_{t+1}^{e s t}-R p_{t}\right]+\right. \\
& \left.A_{2}\left[(1+g-R) p_{t}-g p_{t-1}+\bar{p}(R-1)\right]+\sigma_{\epsilon} \epsilon_{t}\right\},
\end{aligned}
$$

where we have set $A_{1}:=n_{1} /\left[a_{1}\left(\sigma_{1}^{2}+\sigma_{y}^{2}\right)\right], A_{2}:=\left(1-n_{1}\right) /\left[a_{2}\left(\sigma_{2}^{2}+\sigma_{y}^{2}\right)\right]$.

Here we specify $p_{t+1}^{\text {est }}$ as an estimate based on full knowledge of the model $(10)^{5}$, including the beliefs and the reaction of the chartists and the market maker, i.e. as the rational expectations prediction ${ }^{6}$ such that $E_{t}\left(p_{t+1}-p_{t+1}^{e s t}\right)=0$. Thus, by taking expectations on both sides of (10) and solving for $p_{t+1}^{e s t}$, one obtains

$$
p_{t+1}^{e s t}=\frac{p_{t}+\mu\left\{A_{1}\left[\bar{p}\left(R-\alpha_{t}\right)-R p_{t}\right]+A_{2}\left[(1+g-R) p_{t}-g p_{t-1}+\bar{p}(R-1)\right]\right\}}{1-\mu A_{1} \alpha_{t}} .
$$

Substitution of (11) into the price setting equation (10) results in the following two-dimensional (noisy) nonlinear discrete-time dynamical system:

$$
\begin{aligned}
p_{t+1} & =\frac{p_{t}+\mu\left\{A_{1}\left[\bar{p}\left(R-\alpha_{t}\right)-R p_{t}\right]+A_{2}\left[(1+g-R) p_{t}-g q_{t}+\bar{p}(R-1)\right]\right\}}{1-\mu A_{1} \alpha_{t}}+\mu \sigma_{\epsilon} \epsilon_{t}, \\
q_{t+1} & =p_{t} .
\end{aligned}
$$

where $\alpha_{t}=\alpha\left(p_{t}\right)$ is specified according to (6).

The following dynamic analysis is carried out by assuming $\sigma_{\epsilon}=0$, thereby focusing on the so-called deterministic skeleton of the dynamic model. ${ }^{7}$

\section{The map and its properties}

The $2-D$ nonlinear map $T:(p, q) \rightarrow\left(p^{\prime}, q^{\prime}\right)$ that drives the deterministic dynamics can be rewritten as

$$
T:\left\{\begin{array}{l}
p^{\prime}=F(p, q):=\frac{p+\mu\left\{A_{1}[(\bar{p}-p) R-\bar{p} \alpha(p)]+A_{2}[(R-1)(\bar{p}-p)+g(p-q)]\right\}}{1-\mu A_{1} \alpha(p)} \\
q^{\prime}=p
\end{array}\right.
$$

\footnotetext{
${ }^{5}$ Here we assume for simplicity that the fundamentalists know or have learnt the model; subsequent research will need to specify $p_{t+1}^{e s t}$ in some more general way, which takes into account how they can learn about the model, including the market maker and the chartist parameters.

${ }^{6}$ Note that if we interpret the present model as one with three heterogeneous belief-types (see the related discussion in section 2.1), this would be precisely the rational expectations prediction of rational traders.

${ }^{7}$ Such terminology is adopted e.g. in Tong (1990, p. 140). Some numerical experiments on the noisy model (12) are provided in section 4.3.
} 
where $\alpha(p)=\exp \left[-(\bar{p}-p)^{2} / s\right]$, and the symbol ' denotes the unit time advancement operator.

In order to better understand the dynamical behavior of the system, we now focus on the main analytical properties of the map (13), namely the stability properties of the (unique) steady state, and an important symmetry property.

\subsection{Steady state and stability analysis}

It can be proven that the unique fixed point $P^{*}:=\left(p^{*}, q^{*}\right)$ of the map (13) is the long-run fundamental price, that is $P^{*}=(\bar{p}, \bar{p})$. See Appendix A. Here we study the local stability of this fixed point, via the location, in the complex plane, of the eigenvalues of the Jacobian matrix evaluated at $P^{*}$ (that we denote by $J(\bar{p}, \bar{p})$ ). This turns out to be ${ }^{8}$

$$
J(\bar{p}, \bar{p})=\left[\begin{array}{cc}
\frac{1+\mu\left[A_{2}(g-r)-A_{1}(1+r)\right]}{1-\mu A_{1}} & -\frac{\mu A_{2} g}{1-\mu A_{1}} \\
1 & 0
\end{array}\right]
$$

Let us denote by $\operatorname{Tr}$ and Det, respectively, the trace and the determinant of the Jacobian matrix at the fixed point and by $\mathcal{P}(z)=z^{2}-\operatorname{Tr} z+$ Det the associated characteristic polynomial. A well known necessary and sufficient condition for the eigenvalues of $J(\bar{p}, \bar{p})$ to be smaller than one in modulus (which implies local asymptotic stability) is given by the following system of inequalities:

$$
\mathcal{P}(1)=1-\operatorname{Tr}+\text { Det }>0, \quad \mathcal{P}(-1)=1+\operatorname{Tr}+\text { Det }>0, \quad \mathcal{P}(0)=1-\text { Det }>0
$$

that can easily be rewritten in terms of the parameters of the model. The first condition in (14) simplifies to $\mu r\left(A_{1}+A_{2}\right) /\left(1-\mu A_{1}\right)>0$, which holds for $\left(1-\mu A_{1}\right)>0$. Therefore, the analysis can be restricted to the case $0<\mu<1 / A_{1}$, and the second and third condition in (14) become, respectively

$$
\begin{gathered}
\mu\left[A_{1}-A_{2} g+\frac{r}{2}\left(A_{1}+A_{2}\right)\right]<1, \\
1-\mu\left(A_{1}+A_{2} g\right)>0 .
\end{gathered}
$$

Condition (15) holds true when $g \geq \frac{r}{2}+\left(1+\frac{r}{2}\right) \frac{A_{1}}{A_{2}}$, whereas in the opposite case it is satisfied only for $\mu<1 /\left[A_{1}-A_{2} g+\frac{r}{2}\left(A_{1}+A_{2}\right)\right]$, and condition (16) is satisfied for $\mu<$ $1 /\left(A_{1}+A_{2} g\right)$. It follows that the stability region $\Omega$ in the plane of the parameters $(g, \mu)$ is the union of the two regions $\Omega_{F}, \Omega_{N}$ such that

$$
\begin{aligned}
& \Omega_{F}:=\left\{(g, \mu): g \leq g_{0}, 0<\mu<\mu_{F}(g)\right\}, \\
& \Omega_{N}:=\left\{(g, \mu): g>g_{0}, 0<\mu<\mu_{N}(g)\right\},
\end{aligned}
$$

where $g_{0}:=\frac{r}{4}\left(\frac{A_{1}}{A_{2}}+1\right)$, and

$$
\mu_{F}(g)=\frac{1}{A_{1}-A_{2} g+\frac{r}{2}\left(A_{1}+A_{2}\right)},
$$

\footnotetext{
${ }^{8}$ Note that the Jacobian at the steady state (and thus the conditions of local asymptotic stability) does not depend upon the parameter $s$, i.e. it is not influenced by fundamentalist 'sensitivity' to mispricing. However, as numerical simulations reveal, this parameter determines a kind of 'stabilizing' or 'destabilizing' effect on the dynamics in presence of an unstable fundamental equilibrium, by affecting the 'scale' of the attractors in the phase-plane (see also section 4.2).
} 


$$
\mu_{N}(g)=\frac{1}{A_{1}+A_{2} g},
$$

In addition, the branches of hyperbola (17) and (18) which bound the stability region $\Omega$ represent, respectively, a Flip-bifurcation curve (where one of the eigenvalues of $J(\bar{p}, \bar{p})$ is equal to -1 , while the other is smaller than one in modulus) and a Neimark-Sacker bifurcation curve (where the two eigenvalues are complex conjugate of modulus equal to one).

In Fig. 1 we draw the stability region on the plane $(g, \mu)$ under the following choice of parameters: $n_{1}=0.6, a_{1}=0.3, a_{2}=0.275, r=0.001, \sigma_{1}^{2}=\sigma_{2}^{2}=2, \sigma_{y}^{2}=0.02 .{ }^{9}$ From the analytical expressions of the bifurcation curves (17) and (18) we can easily see how the various parameters affect the stability region. For instance, the stability region is enlarged if - ceteris paribus - the risk aversion coefficients $a_{1}$ or $a_{2}$ are increased (see the dotted and dashed boundaries in Fig. 1); a similar effect would result from assuming larger second moment beliefs $\sigma_{1}^{2}$ or $\sigma_{2}^{2}$. In other words, stronger risk perceptions and risk aversion raise the Neimark-Sacker bifurcation threshold for the extrapolation parameter $g$ - denote it by $g_{N}(\mu)$ - for any given price reaction level $\mu$. A similar property holds when chartists behave as contrarians. The stability region is thus enlarged, as visualized by the arrows in Fig. 1. On the contrary, increasing the proportion of the chartists $n_{2}=1-n_{1}$, does not simply result in a downward shifting of the bifurcation curves, but the effect depends in general on the values assigned to the other parameters, as can be checked. ${ }^{10}$

\subsection{Symmetry property}

It is also worth noting that the map $T$ defined by (13) is symmetric with respect to the fixed point $P^{*}=(\bar{p}, \bar{p})$, since symmetric points (with respect to $P^{*}$ ) are mapped into symmetric points under iteration of $T$. As a consequence, a cycle of $T$ is either symmetric with respect to $P^{*}$, or it admits a symmetric cycle, which may give rise to coexistence of attractors. In particular, if a cycle of odd period exists, then the symmetry property implies that a symmetric cycle of the same period must exist too, with the same stability property. If we denote by $S:(p, q) \rightarrow\left(p^{(S)}, q^{(S)}\right)$ the symmetry with respect to $P^{*}=(\bar{p}, \bar{p})$, that is

$$
S:\left\{\begin{array}{l}
p^{(S)}=2 \bar{p}-p \\
q^{(S)}=2 \bar{p}-q
\end{array}\right.
$$

then the symmetry property can be stated as $T(S(p, q))=S(T(p, q)), \forall(p, q)$, or componentwise

$$
\begin{aligned}
& \left(p^{(S)}\right)^{\prime}=2 \bar{p}-F(p, q) \\
& \left(q^{(S)}\right)^{\prime}=2 \bar{p}-p
\end{aligned}
$$

where $F(p, q)$ is defined by the first equation in (13). The proof is provided in Appendix $B$.

\footnotetext{
${ }^{9}$ Under this parameter choice the (positive) quantity $g_{0}:=\frac{r}{4}\left(\frac{A_{1}}{A_{2}}+1\right)$ is very small $\left(g_{0}=0.00059375\right)$ so that the regions $\Omega_{F}$ and $\Omega_{N}$ correspond, roughly speaking, to the regions on the left and on the right of the vertical axis, respectively.

${ }^{10}$ For instance, in the case of the Neimark-Sacker curve, the bifurcation value $g_{N}(\mu)$ increases with $n_{1}$ only if $\mu$ is small enough $\left(\mu<a_{1}\left(\sigma_{1}^{2}+\sigma_{y}^{2}\right)\right)$, while in the opposite case $g_{N}(\mu)$ is a decreasing function of $n_{1}$.
} 


\section{Numerical simulation of the dynamic behavior}

This section is devoted to the numerical exploration of the dynamics of the model, in parameter regions such that the fundamental equilibrium is not locally stable. Bifurcation analysis will be conducted mainly with respect to the chartist parameter $g$. The phase space transitions and the bifurcations that will be described numerically and graphically are global phenomena, in the sense that they could not be detected by the mere analysis of the linearized system around $P^{*}$. Overall, we will focus on situations of coexisting attractors, which are quite often met both in the case of contrarian and of trend chasing chartist behavior. Coexisting attractors determine the possibility that differences in the initial price behavior $\left(p_{0}, q_{0}\right)$ result in qualitatively different asymptotic dynamics of the price. Moreover, they might be associated with random switching among different price and return patterns under the noisy version of the model ${ }^{11}$. We illustrate these phenomena via bifurcation diagrams in the parameter plane $(g, \mu)$, and via phase-plots in the plane $(p, q)$ or representations of the price in the time domain, under different choices of $g$ and $\mu$. The remaining parameters are set as follows:

\begin{tabular}{|c|c|c|c|c|c|c|c|c|}
\hline $\bar{p}$ & $s$ & $n_{1}$ & $a_{1}$ & $a_{2}$ & $R$ & $\sigma_{1}^{2}$ & $\sigma_{2}^{2}$ & $\sigma_{y}^{2}$ \\
\hline 100 & 3 & 0.6 & 0.3 & 0.275 & 1.001 & 2 & 2 & 0.02 \\
\hline
\end{tabular}

\subsection{Dynamics in presence of contrarians}

We consider the case $g<0$ first, in which chartists behave as contrarians and stability is lost via Flip-bifurcation, when $|g|$ becomes large enough (see Fig. 1). The two-parameter bifurcation plot in Fig. $2 a$ indicates with different greytones - in the plane of parameters $(g, \mu), 0>g>-2,0<\mu<1$ - different kinds of asymptotic behavior of the system. The arrow represents a possible "bifurcation path", along which the contrarian parameter $g$ (negative) is increased in magnitude, while the price reaction parameter $\mu$ is kept fixed at $\mu=0.65$. The area on the right of the Flip-bifurcation curve $\mu=\mu_{F}(g)$ is a portion of the stability region, whereas in the grey region just beyond the bifurcation curve two symmetric periodic orbits of period-2 coexist in the phase-space. As a matter of fact - though it can't be seen from the picture - a very narrow strip between the two regions also exists, where the system reaches a stable 2 -cycle, born via a Flip bifurcation at $g \simeq-0.76035 .{ }^{12}$. This attracting 2-cycle in its turn becomes unstable just after a small increase of the parameter, via a pitchfork bifurcation, which produces the coexistence of two stable periodic orbits of period-2 (grey region of Fig. 2a). The coexisting periodic orbits are represented in Fig. $2 b$ together with their basins of attraction. Note that the two attractors are associated with qualitatively different dynamic behaviour, namely prices may be fluctuating below or above the fundamental in the long-run, on average, depending on the initial condition (Fig. 2c). A "reverse" pitchfork bifurcation occurring at $g \simeq-1.25136$ then restores a unique attracting 2-cycle (light grey region of Fig. 2a). Finally, in the black region (upper left corner of Fig. $2 a)$ the generic trajectory is divergent.

\footnotetext{
${ }^{11}$ See Gaunersdorfer, Hommes and Wagener (2003) for an interesting example.

${ }^{12}$ A stable 2-cycle can be numerically detected, for instance, just outside the stability region for $g=$ -0.76140 .
} 


\subsection{Dynamics in presence of trend followers}

Let us now consider the case in which chartists behave as trend followers, $g>0$, and stability is lost via Neimark-Sacker bifurcation for $g$ large enough. As often occurs, the dynamics of the system beyond the Neimark-Sacker bifurcation boundary in the parameter space may be very complicated, and may include cycles of different period and chaos. The two-parameter bifurcation plot in Fig. $3 a$ uses different greytones to partition the parameter plane $(g, \mu)$, $0<g<2,0.5<\mu<1$ in terms of the qualitative asymptotic behavior of the system. The arrow represents a possible bifurcation path along which the extrapolation parameter $g$ (positive) is increased, while the price reaction parameter $\mu$ is kept fixed at $\mu=0.8$. The area on the left of the Neimark-Sacker curve $\mu=\mu_{N}(g)$ is a portion of the stability region, while the dark area in the upper right corner is associated with divergence. The areas with different greytones are Arnol'd tongues ${ }^{13}$ of different period, emanating from the Neimark-Sacker curve. Parameter points in the white area may correspond to high-period cycles, quasiperiodic motion on a closed invariant curve, or chaotic motion. As soon as the Neimark-Sacker curve is crossed, quasiperiodic motion on an attracting invariant closed curve can be observed. The curve changes its shape and increases in size for increasing values of $g$. Coexistence of attractors may be detected when a point in the parameter space is near to the border of an Arnol'd tongue. Consider for instance the region (indicated as '6-cycle') which represents the Arnol'd tongue of a stable period-6 orbit. Close to the left border, the cycle coexists with an attracting closed curve, represented in Fig. $3 b$ together with the coexisting basins of attraction. The boundary of the basin of the 6-cycle is given by the stable manifold of a saddle cycle of period 6, which is born together with the attracting cycle, via saddle-node bifurcation. The attracting curve ceases to exist for higher values of $g^{14}$, so that the 6-cycle remains the unique attractor for some range of the parameter, but close to the left border of the same tongue a new attracting closed curve appears. As a matter of fact, both the stable 6-cycle and its basin of attraction are now inside the area bounded by the new closed attracting curve (see Fig. 3d). Without going into details about the underlying bifurcation mechanisms, we remark that the transition between an attracting 6-cycle external to a closed invariant curve (Fig. 3b), and an attracting 6-cycle internal to a wider closed invariant curve (Fig. 3d) is in fact a complicated sequence of multiple global bifurcations (with first and second homoclinic tangles), as qualitatively described in Agliari et al. (2005) and Agliari et al. (2006). Such phenomena are also closely related to the homoclinic tangles of saddle 4-cycles reported in Brock and Hommes (1997). The 'starfish' shape of the outer curve is related to the stable set of the saddle 6-cycle, which plays the role

\footnotetext{
${ }^{13}$ Arnol'd Tongues are regions in a two-dimensional parameter plane associated with stable $k$-cycles. In smooth dynamical systems they are regions issuing from a Hopf bifurcation curve for flows and from a NeimarkSacker bifurcation curve for maps, in points representing rational rotation numbers $m / k$. The boundaries of an $m / k$ tongue issuing from a Neimark-Sacker bifurcation curve are saddle-node bifurcation curves of a pair of $k$-cycles, and inside the tongue an attracting closed invariant curve may exist, saddle-node connection of the $k$-cycle. Between two tongues issuing from a Neimark-Sacker bifurcation curve of periodicity $m_{1} / k_{1}$ and $m_{2} / k_{2}$ a particular summation rule applies, and also the tongue with periodicity $\left(m_{1}+m_{2}\right) /\left(k_{1}+k_{2}\right)$ exists. In noninvertible maps, overlapping of tongues may occur, leading to multistability. See Boyland (1986) for more details.

${ }^{14}$ It disappears via a "contact bifurcation" due to a contact with the basin boundary of the attracting 6-cycle.
} 
of basin boundary of the inner attracting periodic orbit. ${ }^{15}$ The starfish shape of the attractor causes fluctuations with irregularly varying amplitude (see the black trajectory in Fig. 3e), suggesting clustered volatility at least in a qualitative sense. Moreover, the coexistence of attractors is itself the source of regimes of fluctuations of different amplitude around the unstable fundamental: this can be seen from the price paths drawn in different greytones in Figs. $3 c$ and $3 e$ (corresponding to the phase plots in Figs. $3 b$ and $3 d$ respectively), generated by initial conditions taken in different basins of attraction.

By increasing $g$ further, a different phenomenon of coexistence can be observed. The tongue labelled as '13-cycle' in Fig. 3a, represents in fact a parameter region for which two symmetric 13-cycles co-exist, as a consequence of the symmetry property (see Section 3.2). These attractors replace the closed invariant curve approximately in the range $1.37488<g<$ 1.44969. Fig. If represents the coexisting cycles, together with their basins of attraction.

The foregoing bifurcation analysis has been carried out by varying two key parameters, under a predefined configuration for the remaining ones. However, we have found (via other simulations not reported) that the phenomena observed are quite robust with respect to alternative parameter settings. In particular, changes of the parameter $s$ do not affect the qualitative shape of the plots, but only 'resize' them to different scales: lower values of $s$ (i.e. higher fundamentalists sensitivity to mispricing) reduce the size of the attractors in Figs. $3 b$ and $3 d$, and the amplitude of fluctuations in Figs. $3 c$ and $3 e$.

\subsection{Stochastic simulations}

In this section we present some numerical experiments concerning the noisy dynamical system (12). Fig. 4 represents the time series and the distribution of the returns $\rho_{t}:=\left(p_{t}-p_{t-1}+\right.$ $\left.y_{t}\right) / p_{t-1}$ corresponding to different parameter constellations, under i.i.d. dividends and noise trader demand processes. Both noise trader demand, $\sigma_{\epsilon} \epsilon_{t}$, and dividends, $y_{t}$, are assumed to be normally distributed, with $\sigma_{\epsilon} \epsilon_{t} \sim \mathcal{N}\left(0, \sigma_{\epsilon}^{2}\right), y_{t} \sim \mathcal{N}\left(\bar{y}, s_{y}^{2}\right)$, and the same sample path for the noise processes is combined with alternative deterministic scenarios. ${ }^{16}$ Figs. $4 a, b$ corresponds to the parameter selection of Figs. 3d,e, with strong trend extrapolation by trend followers and coexistence of attractors in the phase space. The deterministic scenario underlying Figs. 4c, $d$ is the one depicted in Figs. 2b, $c$, where chartists behave as contrarians and two different periodic orbits coexist, above and below the unstable fundamental price. Finally, Figs. $4 e, f$ are obtained again for $g>0$, but under weaker trend extrapolation and price reaction than Figs. 4a,b, "inside" the stability domain in the parameter space. While in the latter case the return distribution looks approximately normal (Fig. $4 f$ ) and the time series resembles a gaussian white noise (Fig. 4e), in the case of strong trend extrapolation the return distribution is leptokurtic (Fig. 4b), while in the case of contrarian beliefs the distribution is bimodal (Fig. $4 d$ ), and in both cases the time series display volatility clustering (Figs. $4 a$ and $4 c$ ). These phenomena may be related to the coexistence of attractors and the structure of the basins of attraction of the 'underlying' deterministic model, though of

\footnotetext{
${ }^{15}$ The geometric shape of the closed curve follows the unstable set of the saddle cycle, which is characterized by wide oscillations around the unstable fixed point. This behavior, which is due to the peculiar analytical form of the map (in particular to the effect of the state-dependent weight $\alpha(p)$ in eq. (13)), can also be observed from the boundary of the basin of the 6-cycle in Fig.3d. The external closed invariant curve lies near such a basin boundary and therefore assumes the particular starfish shape of Fig.3d.

${ }^{16}$ Note that the average dividend of the simulation is consistent with agents' expected dividend $\bar{y}:=\bar{p}(R-1)$.
} 
course the connection with the 'deterministic skeleton' represents only a naïve way of looking at the behavior of the noisy model and doesn't help to provide results about the existence of a unique limit distribution of prices and returns - which can be thought of as the appropriate 'equilibrium' notion for such models (see e.g. Böhm and Chiarella (2005), Föllmer, Horst and Kirman (2005)).

\section{Conclusions}

We have developed a two-dimensional discrete-time model of asset price dynamics driven by evolving heterogeneous expectations of two groups of agents, chartists (trend followers or contrarians) and fundamental traders, under a simple market maker price setting rule. A distinguishing feature with respect to earlier contributions is one by which the fundamental traders possess information about their economic environment (including chartists beliefs) and use this in forming expectations about future prices, nevertheless they put increasing weight on a reversion to fundamental as price deviations from equilibrium become larger. Although this mechanism represents the only nonlinear element within this model, it is able to keep the model globally stable when the fundamental equilibrium is locally unstable, and so avoids divergent price paths and replaces them by long-run oscillatory behavior along different, often coexisting attractors, over a wide subset of the parameter space.

Further work on this model needs to be done, in particular the incorporation of timevarying proportions due to switching of traders from chartism to fundamentalism as a consequence of the growing belief of a reversion to the fundamental (along the lines of ChiarellaKhomin (2000)), and the explicit introduction of learning schemes which capture the way fundamental traders can learn about the model structure, including chartists expectations. Furthermore, a more thorough analysis of the noisy model (12) is needed, in particular with regard to the properties of the log-run distributions of prices and returns, under qualitatively different settings for the key behavioural parameters.

\section{Acknowledgements}

This work has been performed with the financial support of MIUR (Italian Ministry of University and Research) within the scope of the national research project (PRIN 2004) "Nonlinear models in economics and finance: interactions, complexity and forecasting". The authors are grateful to participants at the conferences MDEF 2006 (Urbino, 21-23 September 2006), WEHIA 2006 (Bologna, 15-17 June 2006), and Complexity 2006 (Aix en Provence, 17-21 May 2006) for remarks and suggestions on earlier drafts of this work, and also wish to acknowledge the helpful comments of two anonymous referees. The usual caveat applies.

\section{Appendix A. Existence and uniqueness of the steady state}

In this Appendix we prove that the fundamental steady state is the unique equilibrium point of the deterministic map (13).

The 'fundamental' $P^{*}:=(\bar{p}, \bar{p})$, where $\bar{p}=\bar{y} /(R-1)$ is easily recognized as a steady state, by simply setting $\left(p^{\prime}, q^{\prime}\right)=(p, q)=(\bar{p}, \bar{p})$, and therefore $\alpha(p)=1$, in (13). To see that no further steady states exist, denote by $\widehat{P}=(\widehat{p}, \widehat{q})=(\widehat{p}, \widehat{p})$ a generic fixed point of (13), possibly different from $P^{*}$. Then $\widehat{P}$ must satisfy

$$
\widehat{p}=\frac{\widehat{p}+\mu\left\{A_{1}[\bar{p}(R-\widehat{\alpha})-R \widehat{p}]+A_{2}[(1+g-R) \widehat{p}-g \widehat{p}+\bar{p}(R-1)]\right\}}{1-\mu A_{1} \widehat{\alpha}},
$$


where $\widehat{\alpha}:=\alpha(\widehat{p})=\exp \left[-(\bar{p}-\widehat{p})^{2} / s\right]$. Condition (22) is easily simplified to:

$$
\mu A_{1} \widehat{\alpha}(\bar{p}-\widehat{p})=\mu(\bar{p}-\widehat{p})\left[A_{1} R+A_{2}(R-1)\right] .
$$

Of course (23) holds for $\widehat{p}=\bar{p}$, while for $\widehat{p} \neq \bar{p}$ it is equivalent to

$$
\alpha(\widehat{p})=R+\frac{A_{2}}{A_{1}}(R-1),
$$

which admits no solutions because $\alpha(\widehat{p}) \in(0,1]$, whereas $R+\frac{A_{2}}{A_{1}}(R-1)>R>1$. It follows that $\widehat{P}=P^{*}$ is the unique fixed point of (13).

\section{Appendix B. Symmetry of the map}

In this Appendix we prove that the symmetry property (20)-(21) holds for any $(p, q)$. Note first that $(21)$ holds in that it can be rewritten as $\left(q^{(S)}\right)^{\prime}=p^{(S)}$, due to (19). Consider now (20), and note that from (19) it follows that: $\left(p^{(S)}-\bar{p}\right)=(\bar{p}-p),\left(p^{(S)}-q^{(S)}\right)=(q-p)$, whereas obviously $\alpha\left(p^{(S)}\right)=\alpha(p)$. Therefore

$$
\begin{aligned}
& F\left(p^{(S)}, q^{(S)}\right)= \\
& =\frac{p^{(S)}+\mu\left\{A_{1}\left[\left(\bar{p}-p^{(S)}\right) R-\bar{p} \alpha\left(p^{(S)}\right)\right]+A_{2}\left[(R-1)\left(\bar{p}-p^{(S)}\right)+g\left(p^{(S)}-q^{(S)}\right)\right]\right\}}{1-\mu A_{1} \alpha\left(p^{(S)}\right)}= \\
& =\frac{2 \bar{p}-p+\mu\left\{A_{1}[(p-\bar{p}) R-\bar{p} \alpha(p)]+A_{2}[(R-1)(p-\bar{p})+g(q-p)]\right\}}{1-\mu A_{1} \alpha(p)}= \\
& =\frac{2 \bar{p}-2 \bar{p} \alpha(p) \mu A_{1}}{1-\mu A_{1} \alpha(p)}-\frac{p+\mu\left\{A_{1}[(\bar{p}-p) R-\bar{p} \alpha(p)]+A_{2}[(R-1)(\bar{p}-p)+g(p-q)]\right\}}{1-\mu A_{1} \alpha(p)}= \\
& =2 \bar{p}-F(p, q) .
\end{aligned}
$$




\section{References}

Agliari, A., Bischi, G.I., Dieci, R. \& Gardini, L. (2005). Global bifurcations of closed invariant curves in two-dimensional maps: a computer assisted study. International Journal of Bifurcation and Chaos, 15, 1285-1328.

Agliari, A., Bischi, G.I. \& Gardini, L. (2006). Some methods for the global analysis of closed invariant curves in two-dimensional maps. (In T. Puu \& I. Sushko (Eds.), Business Cycle Dynamics: Models and Tools (pp. 7-47). Berlin: Springer.)

Boyland, P.L. (1986). Bifurcations of circle maps: Arnold Tongues, bistability and rotation intervals. Communications in Mathematical Physics, 106, 353-381.

Böhm, V. \& Chiarella, C. (2005). Mean variance preferences, expectations formation and the dynamics of random asset prices. Mathematical Finance, 15, 61-97.

Brock, W. \& Hommes, C.H. (1997). A rational route to randomness. Econometrica, 65, 1059-1095.

Brock, W. \& Hommes, C.H. (1998). Heterogeneous beliefs and routes to chaos in a simple asset pricing model. Journal of Economic Dynamics and Control, 22, 1235-1274.

Chiarella, C. (1992). The dynamics of speculative behaviour. Annals of Operations Research, 37, 101-123.

Chiarella, C. \& He, X.-Z. (2001). Heterogeneous beliefs, risk and learning in a simple asset pricing model. Computational Economics, 19, 95-132.

Chiarella, C. \& He, X.-Z. (2003). Heterogeneous beliefs, risk and learning in a simple asset pricing model with a market maker. Macroeconomic Dynamics, 7, 503-536.

Chiarella C. \& Khomin, A. (2000). The dynamic interaction of rational fundamentalists and trend chasing chartists in a monetary economy. (In D. Delli Gatti, M. Gallegati \& A. Kirman (Eds.), Interaction and Market Structure: Essays on Heterogeneity in Economics (pp. 151-165). Berlin: Springer.)

Day, R.H. \& Huang, W. (1990). Bulls, bears and market sheep. Journal of Economic Behavior and Organization, 14, 299-329.

De Grauwe, P., Dewachter, H. \& Embrechts, M. (1993). Exchange rate theories. Chaotic models of the foreign exchange market. Oxford: Blackwell.

Föllmer, H., Horst, U. \& Kirman, A. (2005). Equilibria in financial markets with heterogeneous agents: a probabilistic perspective. Journal of Mathematical Economics, 41, $123-155$.

Gaunersdorfer, A., Hommes, C.H. \& Wagener, F. (2003). Bifurcation routes to volatility clustering under evolutionary learning. Technical Report No. 03-03, CeNDEF, University of Amsterdam.

Hommes, C.H., Huang, H. \& Wang, D. (2005). A robust rational route to randomness in a simple asset pricing model. Journal of Economic Dynamics and Control, 29, 1043-1072.

Hommes, C.H. (2006). Heterogeneous agent models in economics and finance. (In K.L. Judd \& L. Tesfatsion (Eds.), Handbook of Computational Economics, Volume 2: AgentBased Computational Economics (pp. 1109-1186). Amsterdam: North-Holland).

LeBaron, B. (2006). Agent-based Computational Finance. (In K.L. Judd \& L. Tesfatsion (Eds.), Handbook of Computational Economics, Volume 2: Agent-Based Computational Economics (pp. 1187-1234). Amsterdam: North-Holland).

Tong, H. (1990). Nonlinear time-series. A dynamical system approach. Oxford: Clarendon Press. 


\section{Figure captions}

Figure 1. Region of local asymptotic stability of the steady state, in the plane of the parameters $g, \mu$, and sensitivity of the bifurcation boundaries to the risk-aversion coefficients.

Figure 2. Deterministic dynamics with fundamentalists and contrarians. (a) Twoparameter bifurcation plot for $0>g>-2,0<\mu<1$. (b) Coexisting attracting cycles of period-2 and their basins of attraction. (c) Two time series starting with initial conditions chosen from different basins of attraction.

Figure 3. Deterministic dynamics with fundamentalists and trend followers. (a) Twoparameter bifurcation plot for $0<g<2,0.5<\mu<1$. (b) An attracting closed curve sharing the phase-plane with an outer attracting orbit of period-6, and (c) two time series starting with initial conditions chosen from the coexisting basins of attraction. (d) An attracting orbit of period- 6 surrounded by a competing "star-fish" attractor, and (e) two time series starting with initial conditions chosen from different basins. (f) Two symmetric coexisting orbits of odd period (period-13) with their basins of attraction.

Figure 4. Dynamic outcome of the noisy model, under the same sample paths for the noise processes, but alternative parameter configurations. (a), (b) Return time series and distribution under strong trend extrapolation $(g=1.1683, \mu=0.8)$. (c), (d) Return time series and distribution in presence of contrarians $(g=-0.85, \mu=0.65)$. (e), (f) Return time series and distribution under weaker trend extrapolation $(g=0.5, \mu=0.5)$. Both dividends and noise traders' demand are i.i.d. normal with standard deviations $s_{y}=0.03$, $\sigma_{\epsilon}=0.8$, respectively. Numerically obtained distributions are based on 6000 observations after excluding a transient of 4000 iterations. 
Figure 1

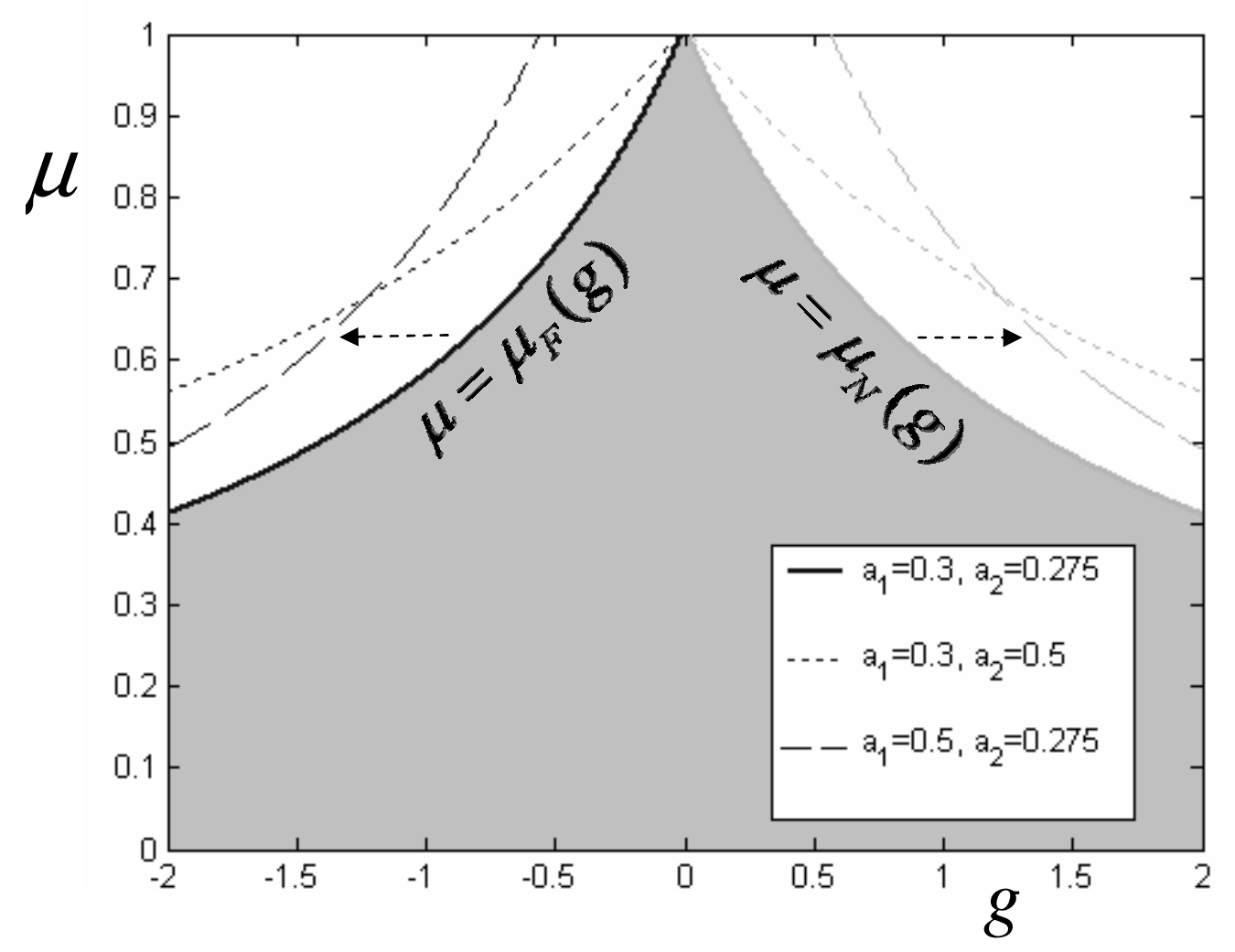




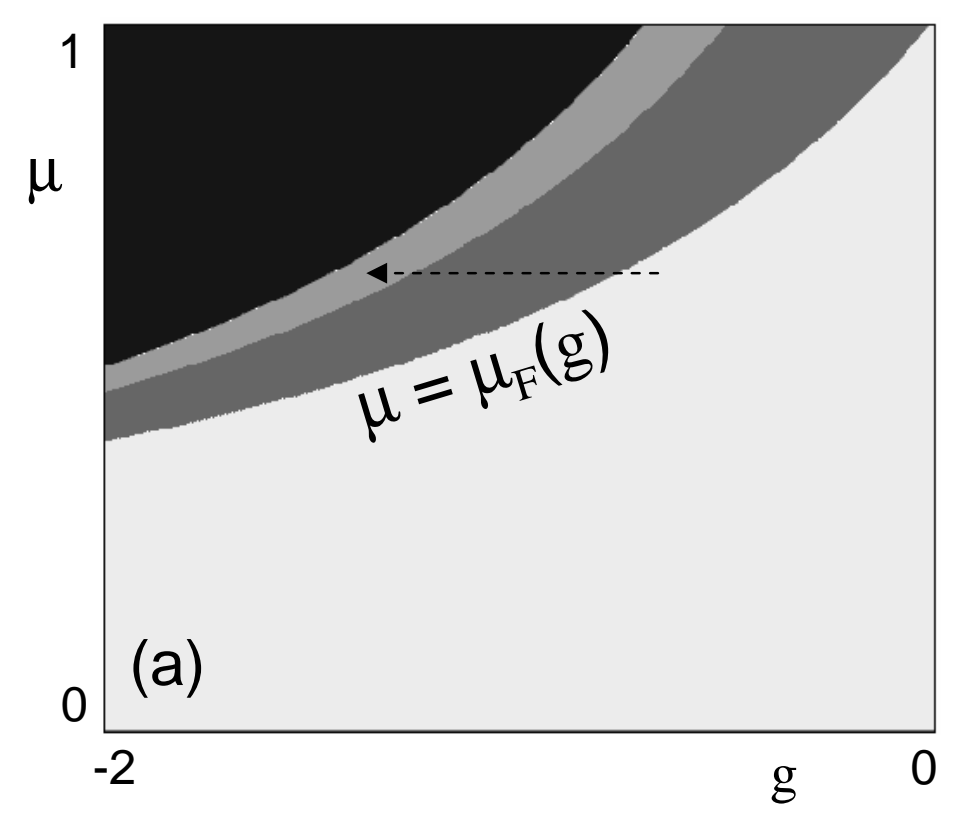

\section{Figure 2}

$\mu=0.65 \quad g=-0.85$
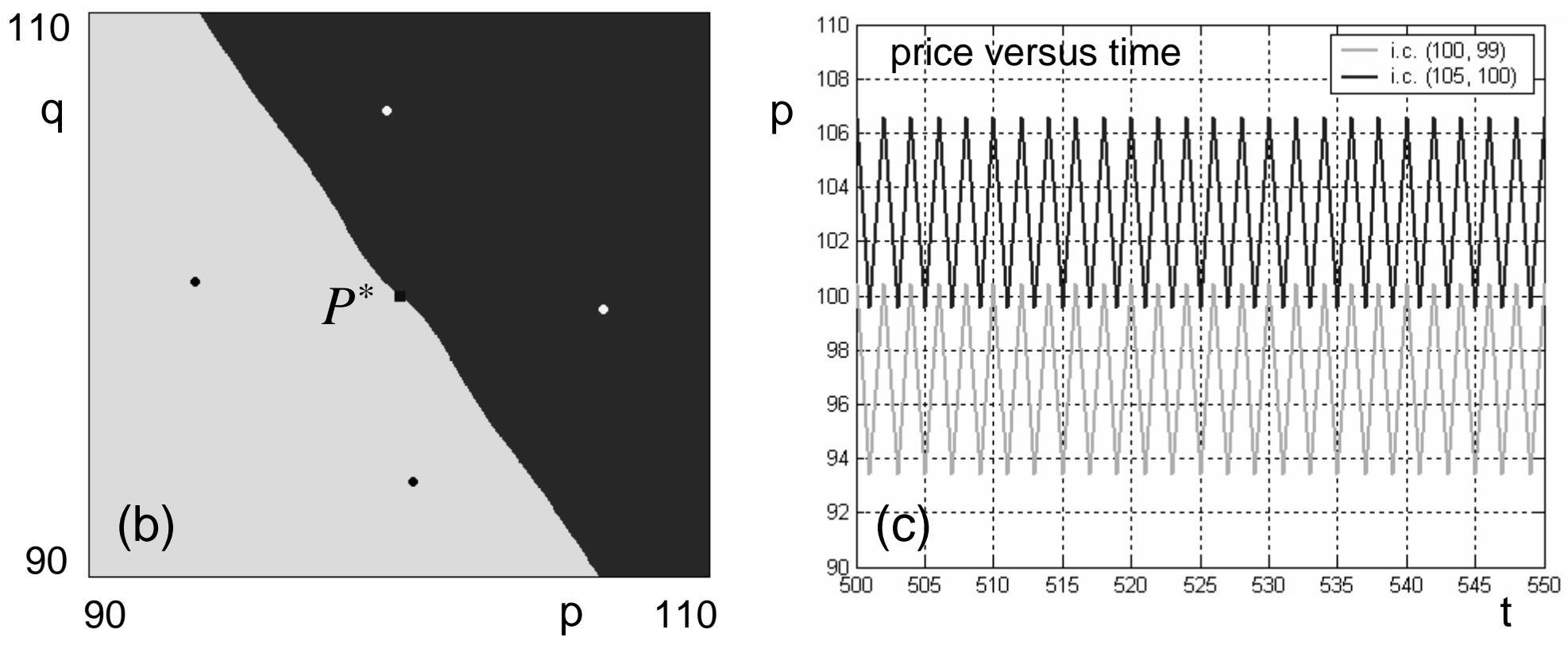
Figure 3
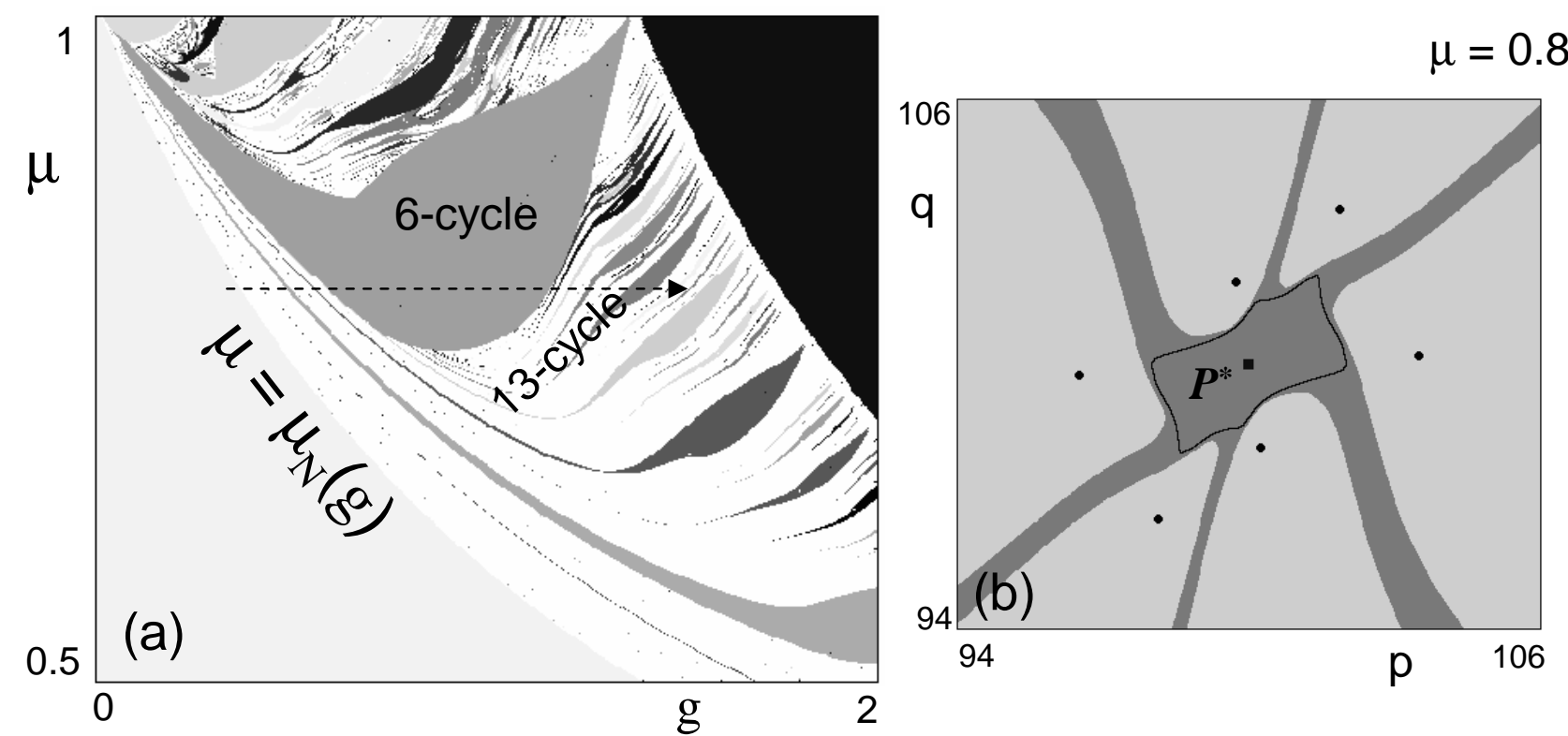

$g=0.6$

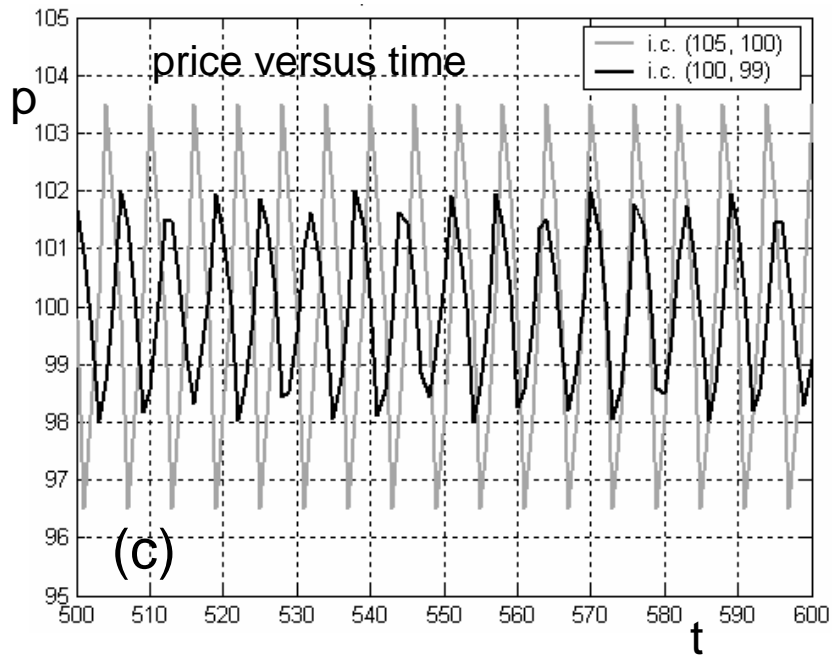

$$
\mu=0.8 \quad g=1.1683
$$
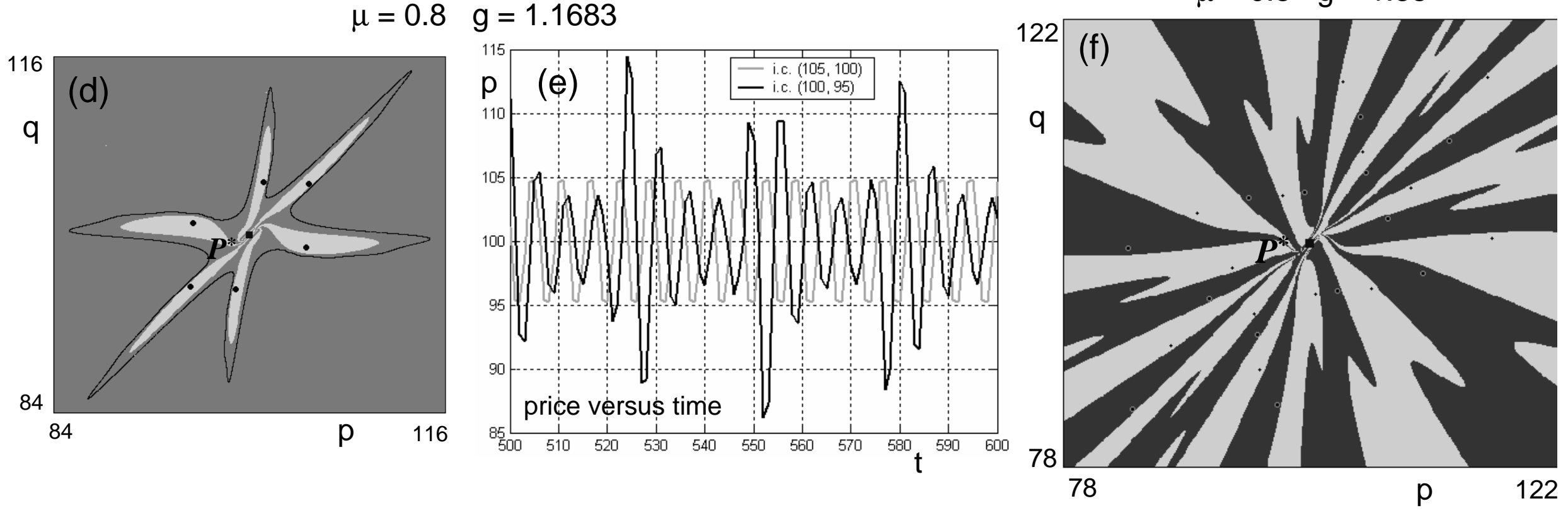
Figure 4
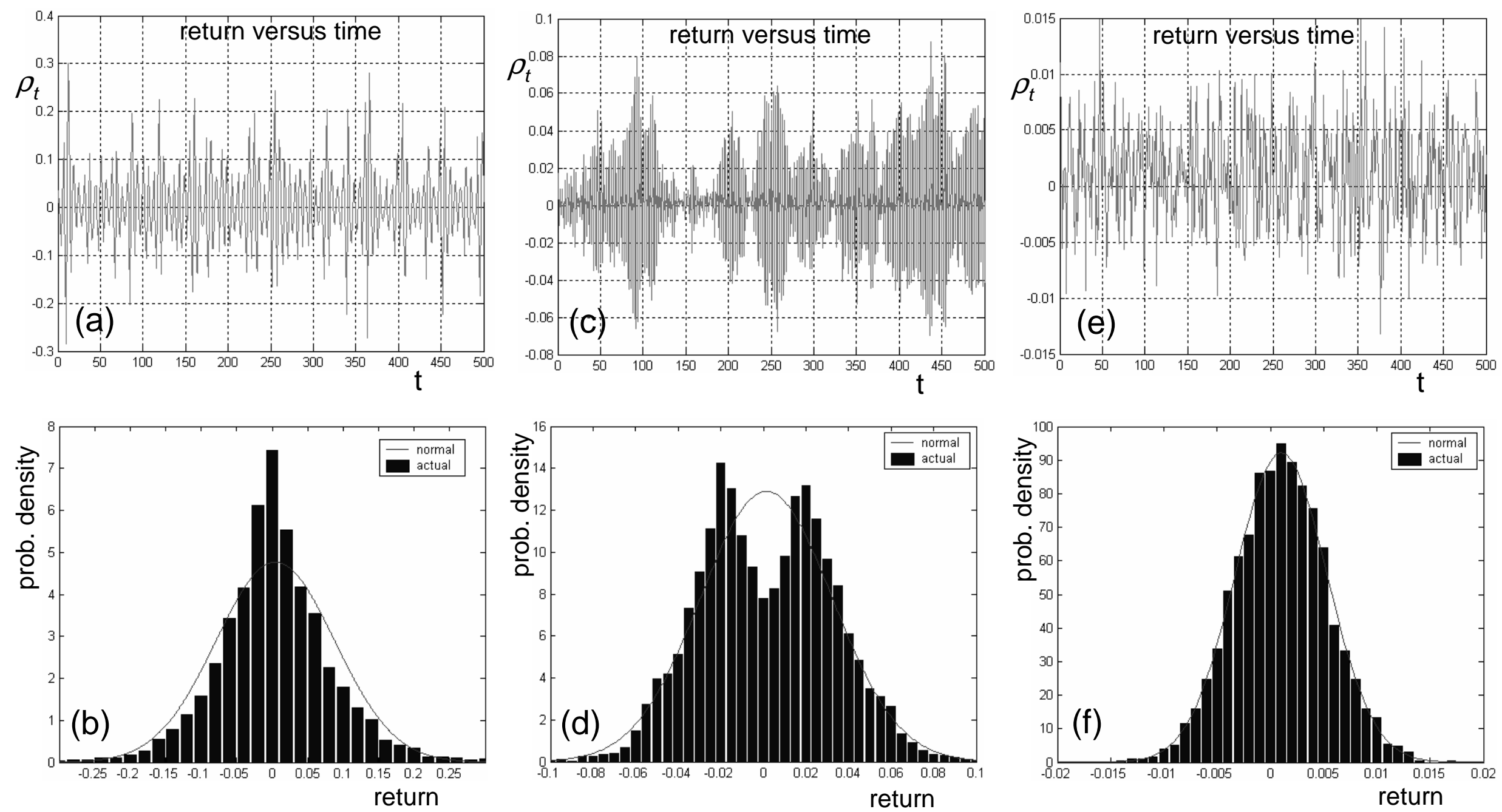